\title{
Minimum Diameter
}

National Cancer Institute

\section{Source}

National Cancer Institute. Minimum Diameter. NCI Thesaurus. Code C120715.

The minimum value in a range of values that describe the diameter of an entity. 